\title{
Postmortem microbiology as a routine tool for legal-medicine in Italy: is it time?
}

\author{
Francesco d'Aleo, ${ }^{1}$ Roberta Bonanno, ${ }^{1}$ Giovanna Bianco, ${ }^{1}$ Antonino Trunfio ${ }^{2}$ \\ ${ }^{1}$ Department of Clinical and Experimental Medicine, "G. Martino" Hospital, University of Messina; ${ }^{2}$ Private Specialist \\ in Legal Medicine, Messina, Italy
}

\begin{abstract}
Summary
Postmortem microbiology may be important to determine the cause of the death. We report a case study of Klebsiella pneumoniae meningitis where the lack of sampling standardization and poor knowledge of this tool could affect medical-legal investigation.
\end{abstract}

\section{Introduction}

Postmortem microbiology is an important tool in legal medicine and forensic pathology, as it helps to determine the manner and cause of the death $(1,4,5)$. The three main indications for its use are: i) a clinically suspected but not confirmed ante-mortem infection; ii) signs of infection at autopsy; iii) as part of a standard protocol in difficult scenarios such as sudden unexpected death. In Europe a high percentage of pathologists recur to postmortem samples for microbiological analysis (8) but in Italy these techniques are not included in standard autopsy protocols. We report a case that shows the

Correspondence: Francesco d'Aleo, Laboratory of Microbiology, Department of Clinical and Experimental Medicine "G. Martino" Hospital, University of Messina, Via Consolare Valeria 1, 98125 Messina (ME), Italy.

E-mail: fdaleo83@gmail.com

Key words: Postmortem microbiology; Klebsiella, meningitis.

Acknowledgements: the authors gratefully thank Prof. Pasquale Urbano, for providing editing.

Contributions: FdA and AT contributed equally to the work.

Conflict of interest: the authors declare no potential conflict of interest.

Received for publication: 2 June 2017.

Revision received: 29 June 2017.

Accepted for publication: 30 June 2017.

(C) Copyright F. d'Aleo et al., 2017

Licensee PAGEPress, Italy

Microbiologia Medica 2017; 32:6828

doi:10.4081/mm.2017.6828

This article is distributed under the terms of the Creative Commons Attribution Noncommercial License (by-nc 4.0) which permits any noncommercial use, distribution, and reproduction in any medium, provided the original author(s) and source are credited. importance of microbiological testing to establish the cause of death. We refer of a Klebsiella pneumoniae meningitis $(7,9)$ diagnosed only after dead with postmortem microbiology tool.

\section{Brief Report}

A woman of 50 years-old was admitted to the emergency room after a car accident. Computerized axial tomography showed a fracture of the left orbit and temporal bone. She was admitted to the general surgical ward where she continued care; on the fifth day her clinical condition worsened, with fever $\left(40.2^{\circ} \mathrm{C}\right)$ and acute respiratory distress syndrome; an empirical antibiotic therapy was started, with Teicoplanin $3 \times 600 \mathrm{mg} / \mathrm{dL}$. Levofloxacin $2 \times 500 \mathrm{mg} / \mathrm{dL}$. Blood and sputum cultures were performed. Unexpectedly, on the following day the patient died. Autopsy was carried out after $24 \mathrm{~h}$, using standard technique (2). Abdominal and thoracic organs did not show pathological findings. The skull was opened and all the brain and cerebellum surfaces appeared covered by a thick purulent exudates; the brain's convolutions were flattened and the sulci filled with pus (Figure 1). The specialist of legal-medicine according to the specialist of microbiology obtained swabs of the purulent exudate and the brain surface. Each swab was plated on selective and nonselective media (Columbia blood agar, Mannitol salt agar, MacConkey agar, Chocolate agar, and Sabouraud agar). All plates were incubated up to seven days under aerobic conditions and for Chocolate agar also under $5 \% \mathrm{CO} 2$ atmosphere at $36 \pm 1{ }^{\circ} \mathrm{C}$. The sole Klebsiella pneumoniae was isolated and identified from all postmortem swabs by exudate and brain's surface; the same organism isolated by the premortem blood and sputum cultures. Antibiotic susceptibility tests, performed by Vitek (bioMérieux, Mercy-L'Etoile, France), indicated the multi-drug resistant (MDR) phenotype for all isolates (Table 1). To detect the production of carbapenemases we used screening it by Carba NP test as recommended in Eucast guideline.

\section{Discussion and Conclusions}

Klebsiella pneumoniae is an important cause of nosocomial infections and it has been recognized as an uncommon pathogen causing metastatic localizations such as meningitis $(3,9)$.

Our aim was to assess the utility of postmortem microbiology for the medical-legal determination of the cause and manner of death. Our case regarded a purulent meningitis diagnosed only after the exitus; its etiology could be determined only by the clinical microbiology lab, activated by the specialist of legal-medicine, because it is indicated in cases of unexpected death. 
Unfortunately in our country diagnostic microbiology is seldom applied to postmortem samples (3), and there are no validated and standardized sampling protocols. As in our case, a microbiological invasion may cause or contribute to rapid death without histological inflammation (5). We have obtained only samples from exudates and brain surface; other possible samples (blood, liver, spleen) should have been taken before starting the autopsy.

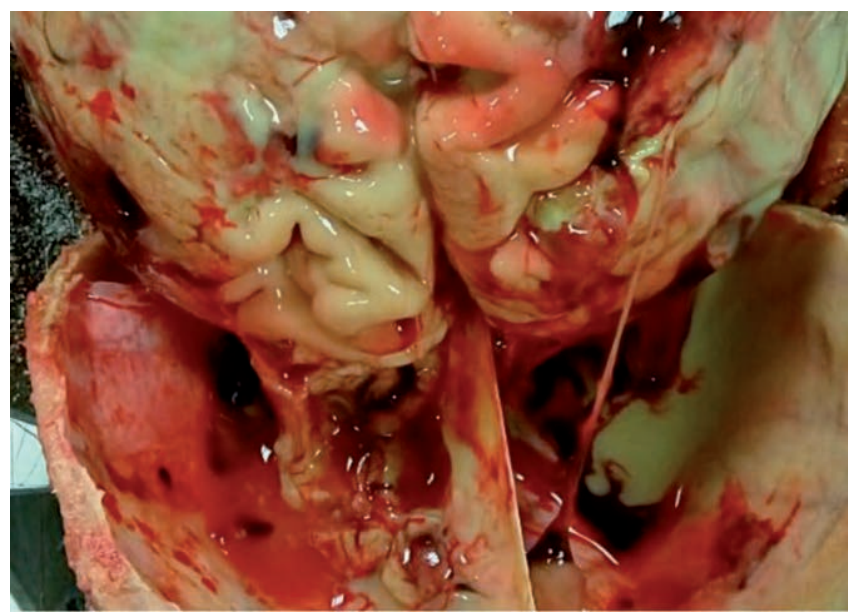

Figure 1. A purulent exudate that covered the hemispheres. The brain's convolutions were flattened and the sulci were filled with pus.

Table 1. Antimicrobial susceptibility testing. MIC values, as assessed by Vitek $\mathbf{2 . 0}$ automated system, were suggestive for susceptibility (S), intermediate susceptibility (I) or resistance (R) according to EUCAST.

\begin{tabular}{lcc} 
Drug & MIC & R/IS \\
Amikacin & $>16$ & $\mathrm{R}$ \\
Amo-Clavulanic & $>32 / 2$ & $\mathrm{R}$ \\
\hline Ampicillin & $>8$ & $\mathrm{R}$ \\
Cefepime & $>8$ & $\mathrm{R}$ \\
\hline Cefotaxime & $>4$ & $\mathrm{R}$ \\
Ceftazidime & $>8$ & $\mathrm{R}$ \\
\hline Cefuroxime & $>8$ & $\mathrm{R}$ \\
Ciprofloxacin & $>1$ & $\mathrm{R}$ \\
\hline Colistin & $\leq 1$ & $\mathrm{~S}$ \\
Ertapenem & $>1$ & $\mathrm{R}$ \\
\hline Fosfomicin & 64 & $\mathrm{R}$ \\
Gentamicin & 4 & $\mathrm{I}$ \\
\hline Imipenem & $>8$ & $\mathrm{R}$ \\
Levofloxacina & $>2$ & $\mathrm{R}$ \\
\hline Meropenem & $>8$ & $\mathrm{R}$ \\
Piperacillin & $>16$ & $\mathrm{R}$ \\
\hline Piperacillin/Taz & $>16 / 4$ & $\mathrm{R}$ \\
Tigeciclin & 2 & $\mathrm{I}$ \\
\hline Tobramicin & $>4$ & $\mathrm{R}$ \\
Trimetoprim/Sulf & $>4 / 76$ & $\mathrm{R}$ \\
\hline & &
\end{tabular}

The ESCMID Study Group for Forensic and Postmortem Microbiology (ESGFOR) has developed a technical protocol that represents a first step towards a consensus in postmortem microbiology procedures (5).

We hope that our report will stimulate a closer relationship between the specialists of legal-medicine and microbiologists, aiming to make postmortem microbiology a notable part of the medical-legal investigations.

\section{References}

1. Caplan MJ, Koontz FP. Cumitech 35 postmortem microbiology. McCurdy BW, ed. Washington DC: American Society for Microbiology; 2001.

2. Collins KA, Hutchins GM. Dissecting the organ block. In: An introduction to autopsy technique. $2^{\text {nd }}$ ed. Washington DC: College of American Pathologists; 2005.

3. D'Ovidio C, Pompilio A, Crocetta V. Fatal sepsis by Klebsiella pneumoniae in a patient with systemic lupus erythematosus: the importance of postmortem microbiological examination for the ex post diagnosis of infection. Int J Legal Med 2015; 129:1097-101.

4. Fernandez-Rodriguez A, Alberola J, Cohen MC. Post-mortem microbiology analysis. Enferm Infecc Microbiol Clin 2013;31: 685-91.

5. Fernández-Rodríguez A, Cohen MC, Lucena J, et al. How to optimise the yield of forensic and clinical post-mortem microbiology with an adequate sampling: a proposal for standardisation. Eur J Clin Microbiol Infect Dis 2015;34:1045-57.

6. Melot B, Colot J, Guerrier G. Bacteremic community-acquired infections due to Klebsiella pneumoniae: clinical and microbiological presentation in New Caledonia, 2008-2013. Int J Infect Dis 2015;41:29-31.

7. Russotto V, Cortegiani A, Graziano G, et al. Bloodstream infections in intensive care unit patients: distribution and antibiotic resistance of bacteria. Infect Drug Resist 2015;8: 287-96.

8. Saegeman V, Cohen MC, Alberola J, et al. How is post-mortem microbiology appraised by pathologists? Results from a practice survey conducted by ESGFOR. Eur J Clin Microbiol Infect Dis 2017 [In Press].

9 Tang LM. Klebsiella pneumoniae meningitis: prognostic factors. Scand J Infect Dis 1994;26:95-102. 